\title{
Sepsis-induced suppression of lung innate immunity is mediated by IRAK-M
}

\author{
Jane C. Deng, ${ }^{1}$ Genhong Cheng, ${ }^{2}$ Michael W. Newstead, ${ }^{3}$ Xianying Zeng, ${ }^{3}$ \\ Koichi Kobayashi, ${ }^{4}$ Richard A. Flavell, 5 and Theodore J. Standiford ${ }^{3}$
}

\begin{abstract}
1Department of Medicine, Division of Pulmonary and Critical Care Medicine, David Geffen School of Medicine at UCLA, Los Angeles, California, USA. '2Department of Microbiology, Immunology and Molecular Genetics, UCLA, Los Angeles, California, USA. ${ }^{3}$ Division of Pulmonary and Critical Care Medicine, Department of Medicine, University of Michigan Medical Center, Ann Arbor, Michigan, USA. ${ }^{4}$ Department of Cancer Immunology and AIDS, Dana-Farber Cancer Institute, Boston, Massachusetts, USA. ${ }^{5}$ Section of Immunobiology, Yale University School of Medicine and Howard Hughes Medical Institute, New Haven, Connecticut, USA.
\end{abstract}

\begin{abstract}
Sepsis results in a state of relative immunosuppression, rendering critically ill patients susceptible to secondary infections and increased mortality. Monocytes isolated from septic patients and experimental animals display a "deactivated" phenotype, characterized by impaired inflammatory and antimicrobial responses, including hyporesponsiveness to LPS. We investigated the role of the LPS/TLR4 axis and its inhibitor, IL-1 receptor-associated kinase-M (IRAK-M), in modulating the immunosuppression of sepsis using a murine model of peritonitis-induced sepsis followed by secondary challenge by intratracheal Pseudomonas aeruginosa. Septic mice demonstrated impaired alveolar macrophage function and increased mortality when challenged with intratracheal Pseudomonas as compared with nonseptic controls. TLR2 and TLR4 expression was unchanged in the lung following sepsis, whereas levels of IRAK-M were upregulated. Macrophages from IRAK-M-deficient septic mice produced higher levels of proinflammatory cytokines ex vivo and greater costimulatory molecule expression in vivo as compared with those of their WT counterparts. Following sepsis and secondary intrapulmonary bacterial challenge, $I R A K-M^{-/}$animals had higher survival rates and improved bacterial clearance from lung and blood compared with WT mice. In addition, increased pulmonary chemokine and inflammatory cytokine production was observed in $I R A K-M^{-/-}$animals, leading to enhanced neutrophil recruitment to airspaces. Collectively, these findings indicate that IRAK-M mediates critical aspects of innate immunity that result in an immunocompromised state during sepsis.
\end{abstract}

\section{Introduction}

Sepsis is a devastating medical condition that is associated with significant morbidity and mortality. The septic state, in addition to eliciting a significant inflammatory response, paradoxically renders the host immunocompromised, thereby resulting in increased susceptibility to secondary infections. Thus, many patients succumb not to the initial septic insult but to secondary nosocomial infections, particularly bacterial pneumonia.

One of the manifestations of sepsis-induced immunosuppression is macrophage dysfunction (1-5). Clearance of bacterial pathogens from the lung is largely dependent upon effective innate immune responses. Macrophages are important innate immune cells and play a critical role in host defense against bacterial pathogens in the lung (6). Alveolar macrophages (AMs) are capable of ingesting and eradicating bacteria that reach the terminal airspaces. When the number of bacteria overwhelms the macrophage's bactericidal capabilities, the ability to mount an effective antimicrobial response requires the cytokine-mediated recruitment of neutrophils. Monocytes isolated from septic patients and experimental animals with sepsis have decreased phagocytic ability, reduced bactericidal activity, and attenuated proinflammatory cytokine production in response to ex vivo LPS stimulation (1-5, 7-11).

Nonstandard abbreviations used: AM, alveolar macrophage; BAL, bronchoalveolar lavage; CLP, cecal ligation and puncture; IP-10, IFN- $\gamma$-inducible protein 10; IRAK, IL-1 receptor-associated kinase; i.t., intratracheal; MIP-2, macrophage inflammatory protein-2; MPO, myeloperoxidase; PAMP, pathogen-associated molecular pattern. Conflict of interest: The authors have declared that no conflict of interest exists. Citation for this article: J. Clin. Invest. 116:2532-2542 (2006). doi:10.1172/JCI28054.
Mechanisms by which the host senses the presence of a pathogen have been the focus of many important recent immunologic studies. TLRs have been found to be a critical family of receptors for recognition of structural components that are unique to pathogens. The microbial components that are recognized by TLRs are referred to as pathogen-associated molecular patterns (PAMPs) and include structures such as LPS, lipoteichoic acid, flagellin, and microbial DNA. TLRs are expressed on a wide variety of cell types, including antigen-presenting cells, endothelial cells, and fibroblasts. In macrophages and other immune cells, ligation of TLRs leads to downstream proinflammatory cytokine production. Prior studies from our laboratory have demonstrated that TNF- $\alpha$ and Th1 cytokines, including IFN- $\gamma$ and IL-12, are critical mediators of innate immunity, particularly against bacterial pathogens in the lung (12-16).

The signaling pathway for LPS ligation of TLR4 has been partially elucidated. At least 2 separate pathways exist, one of which is dependent on the adaptor protein MyD88 and another that is MyD88 independent. The MyD88-dependent pathway in macrophages leads to the recruitment of IL-1 receptor-associated kinase-1 (IRAK-1) and IRAK-4 to the Toll/IL-1 signaling domain, resulting in phosphorylation and activation of these IRAK proteins. IRAK- 1 and -4 then form a complex with TRAF6, which in turn results in MAPK activation and NF- $\kappa$ B activation. Proinflammatory cytokine transcription is regulated primarily by $\mathrm{NF}-\kappa \mathrm{B}$ activity, although other transcription factors, including AP-1, which is downstream of MAPKs, may also play a role.

Regulation of the TLR4 signaling pathway has also been of significant interest. Several negative regulators of the TLR4 signaling pathway have been identified. One inhibitor in particular, 

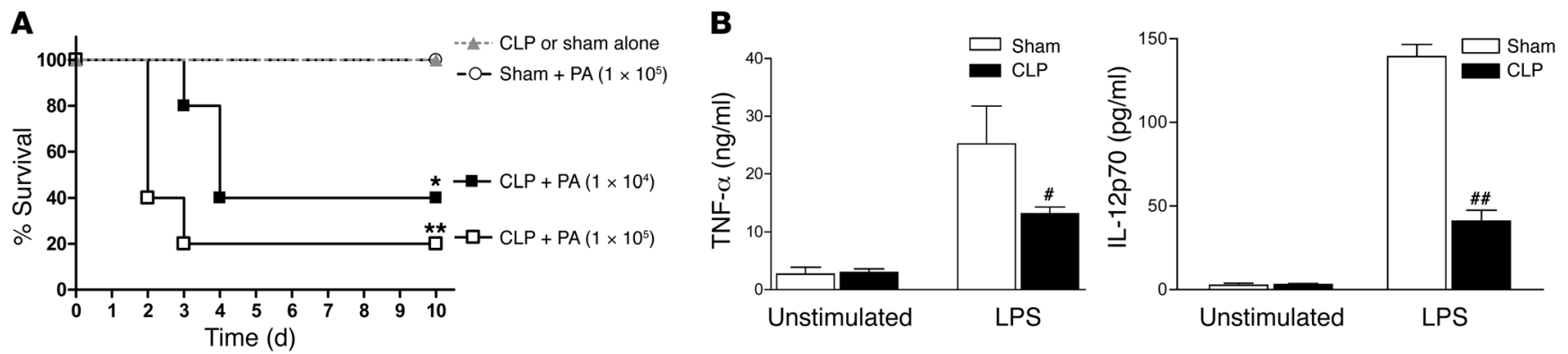

Figure 1

Effect of abdominal sepsis on survival following secondary i.t. Pseudomonas challenge (A) and AM cytokine production in response to LPS (B). (A) Wild-type C57BL/6 mice underwent either CLP with a 26-gauge needle or sham surgery. Twenty-four hours later, mice were administered i.t. $P$. aeruginosa (PA) at the indicated doses and monitored for 10 days after challenge for survival. ${ }^{\star} P<0.01 ;{ }^{* \star} P<0.001$ compared with CLP; $n=10 /$ group; data representative of experiments performed in duplicate. (B) At 24 hours following CLP or sham surgery, mice were sacrificed for the isolation of AMs by BAL. AMs were adherence purified and then stimulated ex vivo with LPS (1 $\mu \mathrm{g} / \mathrm{ml})$ in media or with media alone (unstimulated) for 16 hours. Cell supernatants were collected for determination of TNF- $\alpha$ and IL-12p70 levels by ELISA. \#P<0.05; \#\# $P<0.01$ as compared with corresponding sham; $n=5$ /group; experiments were performed in duplicate.

IRAK-M, appears to be expressed uniquely by monocyte and macrophage populations $(17,18)$. In contrast to IRAK-1 and IRAK-4, IRAK-M lacks kinase activity and negatively regulates signaling through MyD88-dependent TLRs, including TLR2, TLR4, and TLR9 $(19,20)$. Bone marrow-derived macrophages isolated from IRAK-M-knockout (IRAK- $\left.M^{-/-}\right)$animals have been shown to have more vigorous proinflammatory cytokine production in response to several TLR ligands as compared with WT macrophages. Furthermore, IRAK- $M^{-/-}$macrophages are relatively resistant to the development of endotoxin tolerance, which is the phenomenon whereby a cell develops reduced LPS responsiveness following repeated exposure to LPS (19).

In prior studies, we observed that AMs isolated from septic mice have decreased responsiveness to ex vivo LPS stimulation $(7,21)$. Studies by others have shown that macrophages from septic patients demonstrate reduced $\mathrm{NF}-\kappa \mathrm{B}$ activity, similar to that observed in endotoxin tolerance (22). Furthermore, it has been observed that monocytes isolated from septic patients, but not controls, exhibit induction of IRAK-M following ex vivo LPS exposure (23). We hypothesized that TLR signaling, including that of TLR4, is impaired in sepsis, thereby inhibiting the ability of the immune system to respond to secondary gram-negative bacterial infections. We therefore investigated whether the negative regulation of the LPS/TLR4 axis by IRAK-M plays a role in mediating sepsis-induced immunosuppression and whether this molecule could be a potential target for reversing the immunosuppressive effects of sepsis.

\section{Figure 2}

\section{Results}

Cecal ligation and puncture results in impaired innate immunity and responsiveness of alveolar macrophages to LPS stimulation. Preliminary studies indicated that in the murine cecal ligation and puncture (CLP) model of peritonitis-induced sepsis, 24 hours was the time point of maximal AM deactivation and impairment of lung innate host defenses. We therefore challenged WT C57BL/6 mice with intratracheal (i.t.) Psendomonas aeruginosa $\left(10^{5} \mathrm{CFU}\right)$ or saline vehicle at 24 hours after sublethal CLP (26-gauge puncture) or sham surgery. No mortality was observed in uninfected
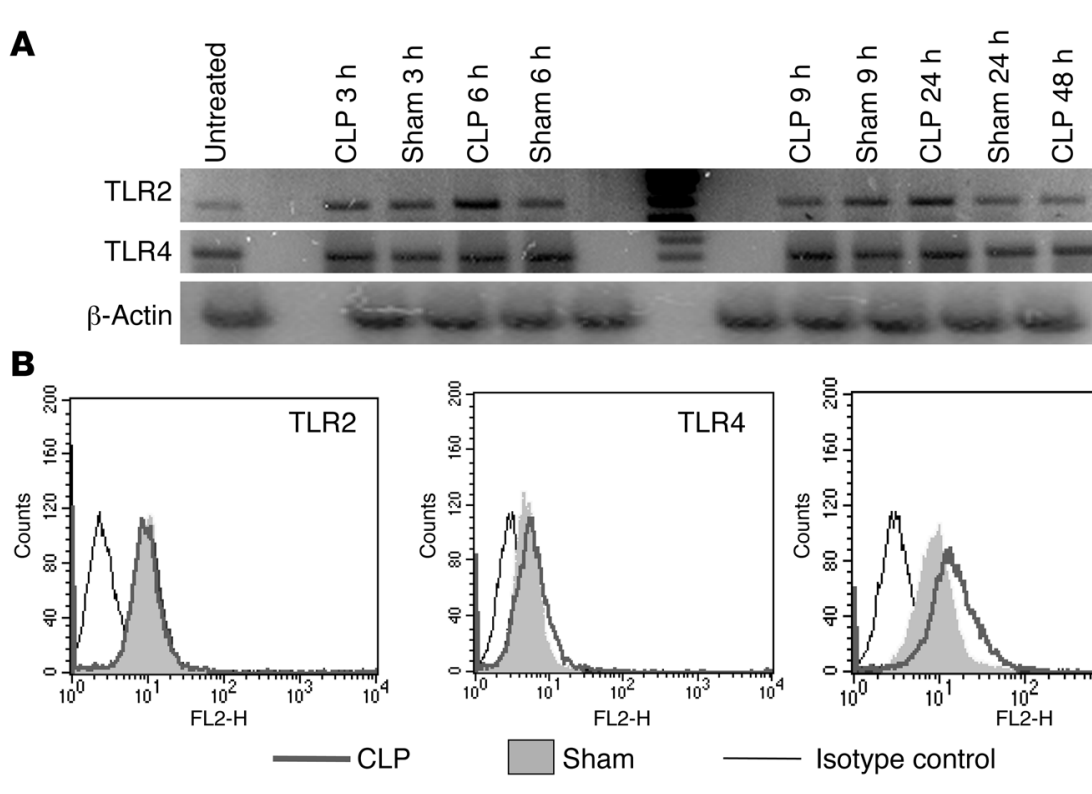

Changes in TLR expression in lung after CLP or sham surgery. (A) Time-dependent expression of TLR2 and TLR4 mRNA in lung after sham surgery or CLP. TLR mRNA was isolated from whole lung at the times noted and mRNA levels determined by RT-PCR. $n=3$ animals/time point combined; experiments were performed in duplicate. (B) Cell-surface expression of TLR2, TLR4, and CD14 by AMs after CLP or sham surgery. At 24 hours after CLP or sham surgery, AMs were isolated by BAL and stained with anti-TLR2, -TLR4, or -CD14 antibodies. Surface expression of these markers was determined by flow cytometry. Histograms are representative of experiments performed in duplicate, with AMs combined from 3 animals per group. 
A

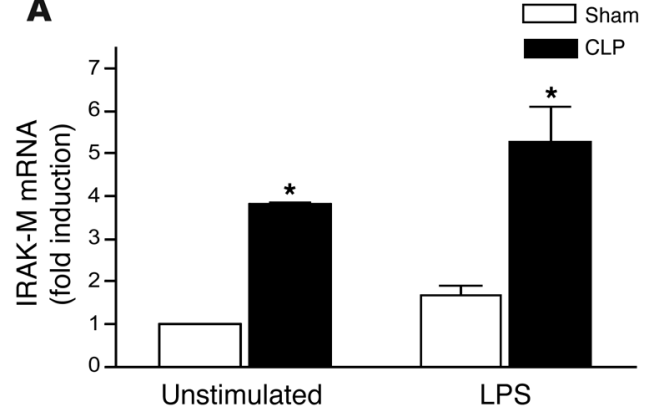

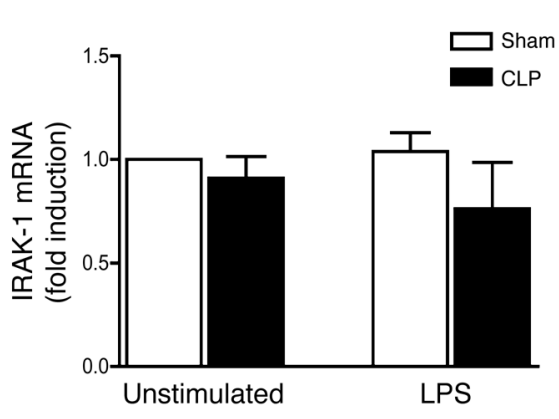

B
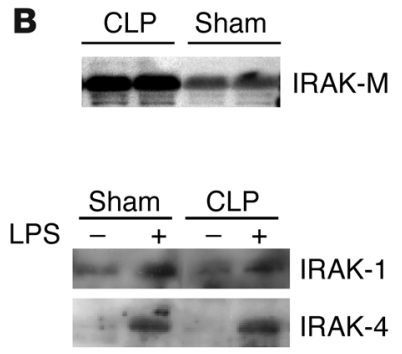

Figure 3

Induction of IRAK-M mRNA (A) or protein (B) in pulmonary macrophages after CLP. At 24 hours after CLP or sham surgery, lungs were harvested and digested with collagenase to obtain single-cell suspensions. Pulmonary macrophages were obtained by adherence purification for 2 hours. The cells were unstimulated or incubated with LPS $(1 \mu \mathrm{g} / \mathrm{ml})$ ex vivo for 6 hours for RNA analysis or incubated with LPS $(1 \mu \mathrm{g} / \mathrm{ml})$ for 16 hours for protein analysis. (A) Expression of IRAK-1 and IRAK-M mRNA was determined by quantitative PCR. Fold increase represents that over expression level from unstimulated macrophages isolated from sham-operated mice. ${ }^{\star} P<0.05$ as compared with sham control. Each condition represents pulmonary macrophages from 3-5 animals combined. (B) Protein levels of IRAK-1, IRAK-4, and IRAK-M were determined by Western immunoblotting.

sham-operated animals, while CLP using a 26-gauge puncture resulted in $0 \%$ mortality (range $0 \%-10 \%$ among experimental replicates), as was anticipated. Sham-operated animals also had $100 \%$ survival following intrapulmonary administration of $10^{5}$ CFU $P$. aeruginosa. In contrast, mice rendered septic by sublethal CLP had substantial mortality when subsequently i.t. challenged with doses of $P$. aeruginosa as low as $10^{4} \mathrm{CFU}$ (Figure 1A). This high lethality was associated with increased $P$. aeruginosa CFU in lung and blood of septic mice, whereas sham-operated mice had minimal or undetectable numbers of bacterial CFU in these compartments by 24 hours following i.t. infection (mean values: $3 \times 10^{8} \mathrm{CFU}$ versus $8 \times 10^{3} \mathrm{CFU}$ [lung, CLP versus sham] and $3 \times 10^{6}$ $\mathrm{CFU} / \mathrm{ml}$ versus $1.2 \mathrm{CFU} / \mathrm{ml}$ [blood, CLP versus sham]; data not shown). Furthermore, adherence-purified AMs isolated from animals 24 hours following CLP surgery had decreased production of the inflammatory cytokines TNF- $\alpha$ and IL-12 in response to ex vivo LPS stimulation as compared with AMs from sham-operated animals (Figure 1B). These studies indicate that CLP results in impaired pulmonary host immune responses to subsequent gram-negative bacterial challenge, and this defect was associated with attenuated AM effector function.

Regulation of pulmonary TLR expression during sepsis. Since TLRs are required for the detection of microbial pathogens, a possible explanation for the increased susceptibility of septic mice to gramnegative bacterial infection in the lung was that sepsis resulted in downregulation of TLR expression. Therefore, we first examined the temporal expression of TLR 4 mRNA in lung during the septic response. No appreciable differences in lung TLR4 mRNA expression were noted between sham and CLP animals. To determine whether selective regulation of individual TLRs occurred, we also examined the expression of TLR2, a key receptor for recognizing cell wall components of gram-positive bacteria and Mycobacterium species. Again, no appreciable differences in the expression of TLR2 message were noted between animals that had undergone sham surgery and CLP at any of the time points examined (Figure 2A).

Given the possibility that differences in TLR expression on individual cell types might not be detected when analyzing whole lung, we next examined TLR expression on the cell surface of AMs. We chose to assess AM TLR expression at 24 hours after sham or CLP surgery, as this was the time point of maximal AM hyporesponsiveness to LPS after induction of sepsis in our earlier studies (data not shown). Furthermore, AMs were studied because these cells are critical initiators of the early innate immune response against invading pathogens in the lung. Both TLR4 and CD14, which are important components of the LPS signaling complex on the surface of macrophages, and TLR2 were expressed at comparable (TLR2 and TLR4) or higher levels (CD14) on AMs lavaged from septic as compared with control sham-operated mice (Figure 2B).

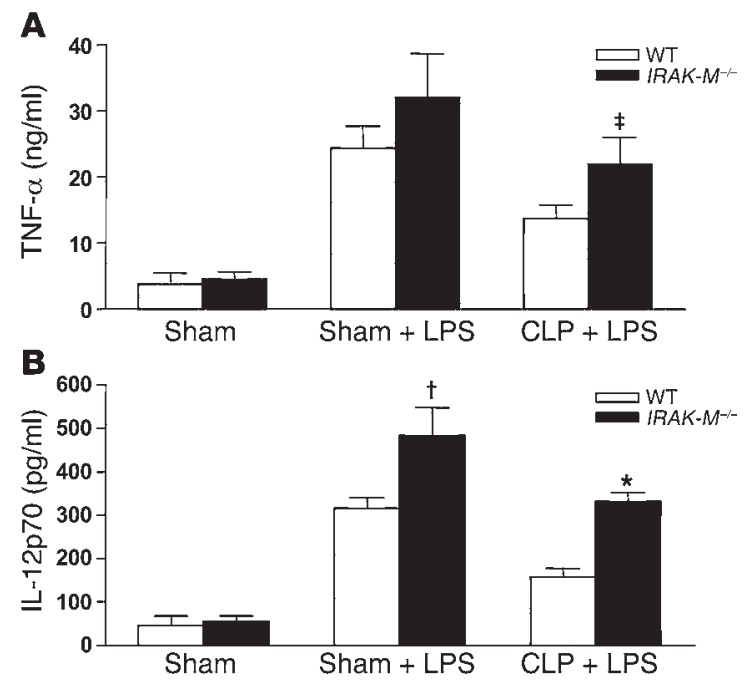

Figure 4

TNF- $\alpha(\mathbf{A})$ and IL-12p70 (B) production by AMs isolated from shamand CLP-operated WT and IRAK-M $M^{--}$mice following ex vivo stimulation with LPS. AMs were isolated from WT and IRAK-M-l- mice 24 hours after either sham surgery or CLP and cultured at a concentration of $5 \times 10^{5} / \mathrm{ml}$ in the presence or absence of LPS $(100 \mathrm{ng} / \mathrm{ml})$ for 16 hours. ${ }^{\star} P<0.05$ and $¥ P=0.05$ compared with cytokine production from LPSstimulated AMs from WT mice isolated 24 hours after CLP. ${ }^{\dagger} P<0.05$ compared with cytokine production from LPS-stimulated AMs from WT mice isolated 24 hours after sham surgery. Data represent combined results from 2 independent experiments; $n=5-16$ per condition. 


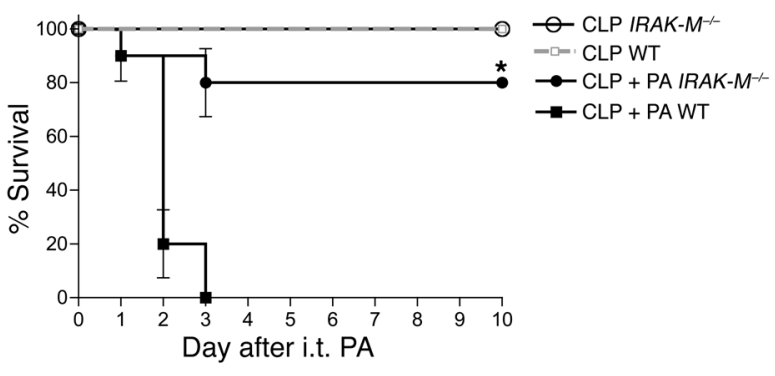

Figure 5

Survival following CLP and i.t. Pseudomonas infection in WT and IRAK-M-1mice. At 24 hours after CLP or sham surgery, WT and IRAK-M-/- mice were i.t. challenged with $P$. aeruginosa $\left(1 \times 10^{5} \mathrm{CFU} / \mathrm{mouse}\right)$. All shamoperated groups had $100 \%$ survival at 10 days (data not shown), as did the groups that underwent CLP alone. Data are representative of experiments performed in triplicate. ${ }^{*} P<0.001$, IRAK- $M^{-1-}$ versus WT after CLP and i.t. Pseudomonas challenge ( $n=10 /$ group).

Hence, the mechanism for decreased ability to mount an effective immune response against pulmonary bacterial infection was not attributable to reduced expression of relevant TLRs by AMs.

IRAK-M expression in pulmonary macrophages following CLP. We next examined expression of downstream regulators of the LPS/TLR4 signaling pathway. As IRAK-1 and IRAK-4 are essential components of signaling through the Toll/IL-1 receptor complex and have been shown to be downregulated in monocyte/macrophage cell lines rendered endotoxin tolerant $(24,25)$, we first examined IRAK-1 and IRAK-4 expression in pulmonary macrophages isolated from septic and control animals. In these experiments, the total population of pulmonary macrophages (alveolar plus interstitial) was isolated from lung digests by adherence purification in order to obtain sufficient number of cells for mRNA and protein analysis. Neither IRAK-1 mRNA nor protein levels were altered in lung macrophages isolated from septic mice as compared with controls (Figure 3, A and B, respectively). Furthermore, the levels of IRAK-4 protein in lung macrophages recovered from sham-operated or CLP mice did not differ (Figure 3B). Subsequent studies were focused on the expression patterns of the TLR signaling inhibitor IRAK-M. We found that CLP resulted in a marked upregulation of both IRAK-M mRNA and protein levels in pulmonary macrophages, particularly following ex vivo LPS stimulation (Figure 3, A and B). Hence, expression of IRAK-M, but not IRAK-1 or IRAK-4, was significantly increased in lung macrophage populations at the time of maximal impairment in macrophage effector function.

Contribution of IRAK-M to altered alveolar macrophage phenotype in sepsis. To assess the contribution of IRAK-M to the sepsis-induced impairment of cytokine production from LPS-stimulated AMs, we harvested AMs by bronchoalveolar lavage (BAL) from WT C57BL/ 6 and IRAK- $M^{-/-}$mice at 24 hours after sham or CLP surgery, then determined IL-12 and TNF- $\alpha$ production ex vivo. The IRAK- $M^{-/-}$mice were generated on a $\mathrm{B} 6$ genetic background, reproduce normally, and are phenotypically indistinguishable from WT B6 mice in the resting state. Unstimulated AMs from sham-operated WT and IRAK-M $\mathrm{M}^{-/-}$ mice produced low levels of TNF- $\alpha$ and IL-12 after 16 hours of culture, and no differences were observed between the 2 strains (Figure 4, A and B). In contrast, LPS stimulation $(100 \mathrm{ng} / \mathrm{ml})$ resulted in a marked increase in TNF- $\alpha$ and IL-12 expression by AMs isolated from sham animals, with higher levels of IL-12 $(P<0.05)$, but not TNF- $\alpha$, in IRAK- $M^{-/-}$cells as compared with WT AMs. Similar to our previous observations, a significant decrease in IL-12 and TNF- $\alpha$ production was noted in WT AMs isolated 24 hours after CLP compared with AMs from nonseptic animals. However, IRAK-M $M^{-/-}$AMs from septic mice produced significantly higher levels of inflammatory cytokines compared with AMs from septic WT animals $(P<0.05)$. We did observe an approximately $30 \%$ reduction in TNF- $\alpha$ and IL-12 production by LPS-stimulated AMs from $I R A K-M^{-/-}$mice after CLP as compared with AMs from sham-operated $I R A K-M^{-/-}$mice, although this difference did not reach the level of statistical significance $(P=0.24$ and $P=0.10$ for TNF- $\alpha$ and IL-12, respectively). Importantly, the production of TNF- $\alpha$ and IL-12 by LPS-treated AMs from IRAK-M-/- mice undergoing CLP was indistinguishable from that by LPS-stimulated AMs from sham WT mice ( $P>0.05$ for both cytokines).

Survival of WT and IRAK- $M^{-1-}$ mice following CLP and subsequent intrapulmonary gram-negative bacterial challenge. To determine whether IRAK-M contributed to the impairment in lung antibacterial responses during sepsis, we performed CLP and sham surgery on IRAK- $M^{-/-}$and WT animals and then challenged animals 24 hours later with i.t. P. aeruginosa $\left(10^{5} \mathrm{CFU}\right)$ to determine 10 -day survival. In initial studies, we found that although IRAK-M ${ }^{-/-}$ animals appeared more ill (exhibiting lethargy, ruffled fur, reduced oral intake) during the first 24 hours following CLP, their survival was not adversely affected compared with that of their WT counterparts following CLP alone. As previously observed, administration of $P$. aeruginosa to WT mice 24 hours after CLP resulted in high mortality. Impressively, $I R A K-M^{-/-}$animals undergoing CLP had markedly increased survival following secondary pulmonary bacterial challenge with $P$. aeruginosa, as compared
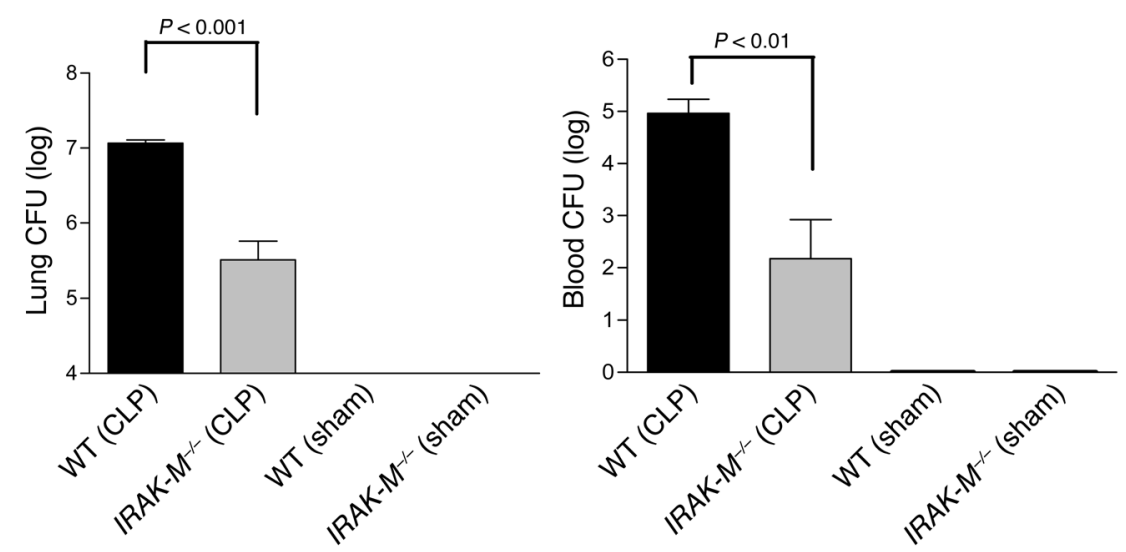

Figure 6

Lung and blood CFU following CLP and i.t. $P$. aeruginosa administration in WT and IRAK- $M^{-/}$ mice. WT and IRAK-M $M^{-/-}$mice underwent CLP or sham surgery on day 0 and i.t. Pseudomonas administration on day 1. Lung and blood were collected for CFU determination 24 hours after administration of bacteria. Both WT and IRAK- $M^{-/-}$sham-operated groups had undetectable bacterial CFU in lung and blood following i.t. Pseudomonas administration. $n=5-6 /$ group; experiments were performed in duplicate. 

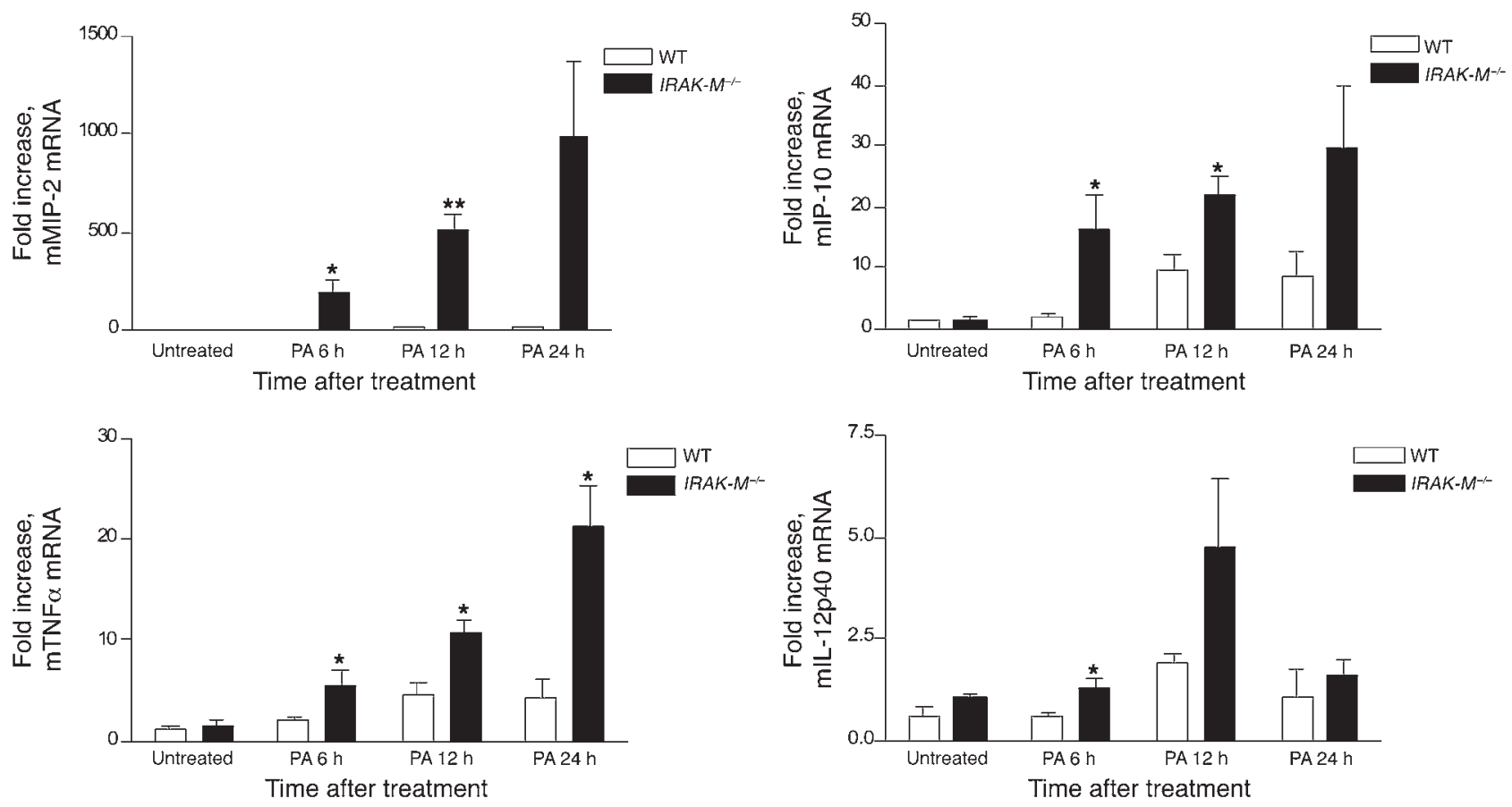

Figure 7

Lung cytokine mRNA expression following CLP and i.t. Pseudomonas challenge in WT and IRAK- $M^{-/-}$mice. WT and IRAK- $M^{-/-}$mice underwent CLP followed 24 hours later by i.t. $P$. aeruginosa administration. At various time points following $P$ seudomonas administration, lungs were harvested for determination of cytokine mRNA levels by real-time quantitative PCR. Data are presented as fold increase in mRNA levels over WT untreated. ${ }^{*} P<0.05 ;{ }^{\star \star} P<0.001 ; n=3-4$ animals/condition combined.

with septic WT mice (Figure 5). Sham-operated mice infected with i.t. Pseudomonas had $100 \%$ survival in both the IRAK- $M^{-/-}$ and WT groups (data not shown).

Bacterial clearance following i.t. P. aeruginosa administration in CLP and sham-operated WT and IRAK-M-1- mice. Experiments were performed to determine whether the increased survival observed in $I R A K-M^{-/-}$animals was due to enhanced bacterial clearance after secondary bacterial challenge. We therefore examined lung and blood bacterial burden in IRAK- $M^{-/-}$and WT mice undergoing CLP followed by i.t. P. aeruginosa challenge. At 24 hours after Pseudomonas administration, septic IRAK- $M^{-/-}$animals had significantly decreased bacterial counts in both the lung and blood, as compared with their WT counterparts (Figure 6). Specifically, IRAK-M $M^{-/-}$mice had approximately 20 - and 40 -fold less P. aeruginosa CFU in their lungs and blood, respectively, than WT mice. Sham-operated controls had undetectable bacterial CFU in both the lung and blood at this time point.

We also assessed for differences in lung histology after $P$. aeruginosa administration. Lung morphology at baseline and at 24 hours following CLP did not differ between WT and IRAK- $M^{-/-}$mice. However, following CLP and i.t. P. aeruginosa administration, we observed large numbers of neutrophils but no free bacteria in the alveoli of IRAK- $\mathrm{M}^{-{ }^{--}}$animals, whereas we found a relative paucity of neutrophils and the presence of considerable numbers of intraalveolar bacteria in WT mice (data not shown).

Lung cytokine production in lung following i.t. P. aeruginosa challenge in septic WT and IRAK-M-/- mice. Previous work from our laboratory has identified the importance of inflammatory cytokines and chemokines (e.g., TNF- $\alpha$, IL-12, and the CXC chemokines macro- phage inflammatory protein-2 [MIP-2] and IFN- $\gamma$-inducible protein 10 [IP-10]) to the innate immune response against bacterial pathogens in the lung $(12-15,26-31)$. We therefore examined

\section{Table 1}

Total lung cytokine levels (pg/lung) in septic WT and IRAK-M ${ }^{-1}$ mice after challenge with $P$. aeruginosa

\begin{tabular}{|c|c|c|c|}
\hline & 0 & $6 \mathrm{~h}$ & $24 \mathrm{~h}$ \\
\hline \multicolumn{4}{|l|}{ TNF- $\alpha$} \\
\hline $\begin{array}{l}\text { WT } \\
\text { IRAK-M } \text { - }^{-}\end{array}$ & $\begin{array}{l}213 \pm 77 \\
241 \pm 81\end{array}$ & $\begin{array}{l}485 \pm 49 \\
885 \pm 113^{A}\end{array}$ & $\begin{array}{l}573 \pm 54 \\
868 \pm 99\end{array}$ \\
\hline \multicolumn{4}{|l|}{ MIP-2 } \\
\hline $\begin{array}{l}\text { WT } \\
\text { IRAK-M-- }\end{array}$ & $\begin{array}{l}74 \pm 13 \\
93 \pm 7\end{array}$ & $\begin{array}{l}242 \pm 80 \\
671 \pm 19^{A}\end{array}$ & $\begin{array}{l}464 \pm 130 \\
721 \pm 240\end{array}$ \\
\hline \multicolumn{4}{|l|}{ IL-12 } \\
\hline $\begin{array}{l}\text { WT } \\
\text { IRAK-M-1- }\end{array}$ & $\begin{array}{l}582 \pm 205 \\
508 \pm 100\end{array}$ & $\begin{array}{c}718 \pm 69 \\
1,353 \pm 211^{A}\end{array}$ & $\begin{array}{r}968 \pm 124 \\
1,690 \pm 145^{A}\end{array}$ \\
\hline \multicolumn{4}{|l|}{ IP-10 } \\
\hline $\begin{array}{l}\text { WT } \\
\text { IRAK-M }{ }^{--}\end{array}$ & $\begin{array}{l}688 \pm 181 \\
958 \pm 112\end{array}$ & $\begin{array}{l}1,135 \pm 30 \\
2,556 \pm 302^{A}\end{array}$ & $\begin{array}{l}1,466 \pm 89 \\
2,056 \pm 167^{A}\end{array}$ \\
\hline \multicolumn{4}{|l|}{ IL-10 } \\
\hline $\begin{array}{l}\text { WT } \\
\text { IRAK-M } \text { - }^{-}\end{array}$ & $\begin{array}{r}2791 \pm 471 \\
3,226 \pm 504\end{array}$ & $\begin{array}{l}5,948 \pm 689 \\
8,135 \pm 1,511\end{array}$ & $\begin{array}{l}4,268 \pm 393 \\
6,784 \pm 783\end{array}$ \\
\hline
\end{tabular}

Data expressed as mean \pm SEM. ${ }^{A} P<0.05$ compared with corresponding WT control. $n=5$ mice per time point. 


\section{Table 2}

BAL cell counts in septic WT and IRAK-M-1- mice after challenge with $P$. aeruginosa

\begin{tabular}{|c|c|c|c|c|}
\hline & Total cell no. & Monocytes & Neutrophils & Lymphocytes \\
\hline \multicolumn{5}{|l|}{$6 \mathrm{~h}$} \\
\hline $\begin{array}{l}\text { WT } \\
\text { IRAK-M-1- }\end{array}$ & $\begin{array}{l}5.2 \times 10^{5} \pm 0.7 \times 10^{5} \\
4.9 \times 10^{5} \pm 0.9 \times 10^{5}\end{array}$ & $\begin{array}{l}5.1 \times 10^{5} \pm 0.7 \times 10^{5} \\
4.5 \times 10^{5} \pm 0.8 \times 10^{5}\end{array}$ & $\begin{array}{l}0.1 \times 10^{4} \pm 0.1 \times 10^{4} \\
3.2 \times 10^{4} \pm 0.2 \times 10^{4}\end{array}$ & $\begin{array}{r}1.0 \times 10^{4} \pm 0.4 \times 10^{4} \\
0.15 \times 10^{4} \pm 0.09 \times 10^{4}\end{array}$ \\
\hline \multicolumn{5}{|l|}{$24 \mathrm{~h}$} \\
\hline $\begin{array}{l}\text { WT } \\
\text { IRAK-M } \text { I-- }^{-}\end{array}$ & $\begin{array}{l}6.3 \times 10^{5} \pm 2.0 \times 10^{5} \\
9.2 \times 10^{5} \pm 1.5 \times 10^{5}\end{array}$ & $\begin{array}{l}5.8 \times 10^{5} \pm 2.1 \times 10^{5} \\
5.1 \times 10^{5} \pm 0.9 \times 10^{5}\end{array}$ & $\begin{array}{c}0.44 \times 10^{5} \pm 0.1 \times 10^{5} \\
4.1 \times 10^{5} \pm 1.6 \times 10^{5 \mathrm{~A}}\end{array}$ & $\begin{array}{l}7.3 \times 10^{3} \pm 2.0 \times 10^{3} \\
2.2 \times 10^{3} \pm 2.2 \times 10^{3}\end{array}$ \\
\hline
\end{tabular}

Data are expressed as absolute cell number \pm SEM. ${ }^{A} P<0.05$ compared with corresponding WT control. $n=5$ mice per time point.

whether IRAK-M modulated the expression of these cytokines in the lungs of CLP animals when they were subsequently challenged with gram-negative bacteria i.t. In these experiments, we performed CLP surgery in WT and IRAK- $M^{-/-}$animals, administered $P$. aeruginosa i.t. 24 hours after surgery, then harvested lungs at 6,12, and 24 hours after Pseudomonas administration to quantitatively examine mRNA expression of selected cytokines and chemokines. As shown in Figure 7, WT CLP mice displayed relatively modest induction of cytokine and chemokine mRNA in the lung ( $<10$-fold) in response to i.t. P. aeruginosa challenge. By comparison, IRAK- $M^{-/-}$mice had significantly higher levels of MIP-2, TNF- $\alpha$, IP-10, and IL-12p40 mRNA, particularly at early time points ( 6 and 12 hours), suggesting that the preservation of cytokine production early in the course of infection is critical to effective lung bacterial clearance and survival in this model. Similarly, we observed significant increases in inflammatory cytokine protein levels in lung homogenates of IRAK-M $M^{-/-}$mice 6 hours after $P$. aeruginosa administration compared with infected WT mice (Table 1). Levels of IL-12 and IP-10 remained elevated in IRAK-M $M^{-/-}$animals out to 24 hours after Pseudomonas administration. Of interest, we also observed a trend toward increased levels of the antiinflammatory cytokine IL-10 in the lungs of $I R A K-M^{-/-}$mice after intrapulmonary bacterial challenge.

Lung leukocyte influx/activation in septic WT and IRAK-M ${ }^{-/-}$mice following i.t. P. aeruginosa administration. The most prominent differences in inflammatory cytokine production between septic $I R A K-M^{-/-}$and WT mice were observed in the levels of cytokines that facilitate lung neutrophil influx, namely MIP-2 and to a lesser extent TNF- $\alpha$. Experiments were performed to characterize the nature of infiltrating leukocytes, including neutrophils, after $P$. aeruginosa administration in septic WT and IRAK-M-1- animals. In initial studies, we observed that cell counts and differentials in the lungs did not differ between WT and IRAK-M $M^{-/-}$mice at baseline or following CLP alone, with more than $95 \%$ of lavaged cells being alveolar macrophages, as determined by morphological criteria (data not shown). We performed CLP surgery on WT and $I R A K-M^{-1-}$ mice, followed 24 hours later by i.t. Pseudomonas administration, then lavaged lungs at 6 and 24 hours after $P$. aeruginosa administration to obtain airspace leukocytes for cell counts and differentials. Importantly, at 24 hours following i.t. Pseudomonas infection, septic IRAK-M $\mathrm{M}^{--}$animals had a nearly 10 -fold increase in the numbers of neutrophils in the lungs compared with infected WT mice (Table 2; $P<0.05$ ). In contrast, we did not observe significant differences in the total numbers of mononuclear cells in the airspaces. We also examined the recruitment and activation of specific lymphocyte and NK cell populations using four-color flow cytometry of leukocyte suspensions obtained from collagenase-digested whole lung. We noted no differences in the number of $\alpha \beta$ T cells $\left(\mathrm{CD}^{+}\right.$or $\left.\mathrm{CD}^{+}\right), \gamma \delta \mathrm{T}$ cells, NK cells, or NKT cells between the WT and IRAK- $M^{-/-}$groups (data not shown), nor did we observe differences in the expression of the activational marker CD69 among any of the cell types examined (data not shown).

In addition to quantitating of neutrophils in BAL fluid, we also measured lung myeloperoxidase (MPO) activity as an indirect measure of the total lung neutrophils present in septic WT and IRAK-M ${ }^{-/-}$ mice after i.t. $P$. aeruginosa administration. As compared with septic WT mice, septic IRAK-M-1- mice had a significantly greater increase in lung MPO activity at both 6 and 24 hours after bacterial challenge (Figure $8 ; P<0.05$ at both time points).

Costimulatory molecule expression by AMs in septic WT and IRAK-M ${ }^{-/-}$ mice following i.t. P. aeruginosa administration. To directly assess the activational status of lung macrophages during infection, we determined the cell-surface expression of the costimulatory molecules CD40, CD80, and CD86 by AMs after CLP and 6 hours after i.t. $P$. aeruginosa administration. In these experiments, AMs were obtained from WT and IRAK-M ${ }^{-/-}$mice by BAL 24 hours after sham surgery or CLP (time 0 ) and 6 hours after i.t. P. aeruginosa administration. We observed no difference in the cell-surface expression of CD40 and CD86 by AMs obtained 24 hours after sham surgery or CLP in WT and $I R A K-M^{-1-}$ mice (Figure 9 and data not shown). However, an increase in CD40 expression, as well as a lesser but significant increase in CD86 expression, was noted in AMs isolated from septic IRAK-M-1- mice 6 hours after i.t. bacterial challenge relative to the considerably blunted response observed in similarly treated WT mice, as indicated by an approximately 2.4 -fold and 1.5 -fold increase in mean fluorescence

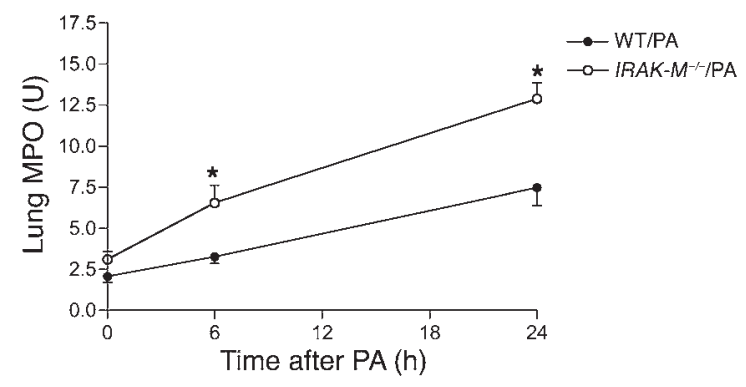

\section{Figure 8}

Total lung MPO activity following CLP and i.t. $P$. aeruginosa challenge in WT and IRAK-M $M^{-/}$mice. $n=3$ animals per group for time $0 ; n=8$ animals per group for the 6 and 24 hour time points. ${ }^{*} P<0.01 \mathrm{com}-$ pared with WT mice. 


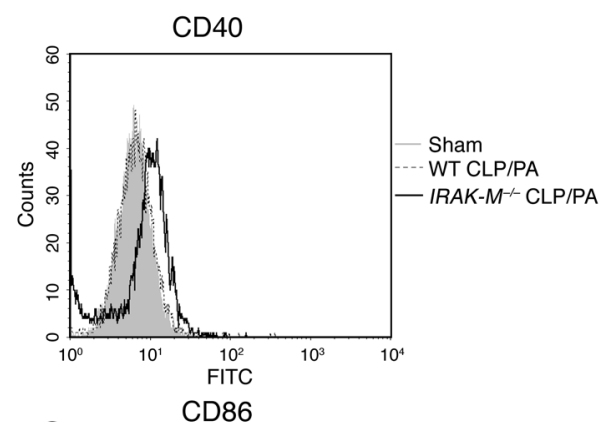

\section{Figure 9}

Cell-surface expression of CD40 and CD86 by AMs after CLP or sham surgery followed by i.t. $P$. aeruginosa administration. At 24 hours after CLP or sham surgery (time 0 ) or 6 hours after $P$. aeruginosa administration, AMs were isolated by BAL and stained with anti-CD40, -CD80, or -CD86 antibodies. Surface expression of these markers was determined by flow cytometry gating on macrophages based on size and surface complexity characteristics. Histograms are representative of experiments performed in duplicate, with AMs combined from 3 animals per group. Shaded histogram represents AMs from mice 24 hours after sham surgery.

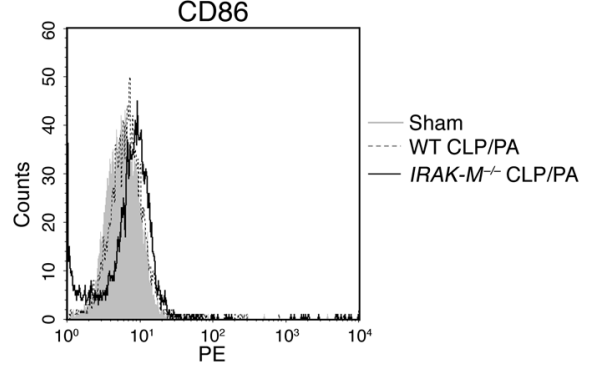

endotoxin tolerant or peptidoglycan tolerant following repeated or prolonged exposure to these TLR ligands. Interestingly, a recent study found that peripheral blood monocytes isolated from septic patients displayed enhanced expression of IRAK-M, as compared with cells isolated from nonseptic controls, highlighting the clinical relevance of this molecule in sepsis (23). Whether the upregulation of IRAK-M during sepsis leads to monocyte hyporesponsiveness to other microbial components, such as flagellin or microbial DNA, remains uncertain.

Alveolar macrophages are central to the generation of effec-

intensity, respectively $(P<0.05$ and $P=0.05$, respectively). No differences in AM CD80 expression were observed between WT and IRAK- $\mathrm{M}^{-/-}$mice 24 hours after sham surgery or CLP or at 6 hours after bacterial challenge (data not shown).

\section{Discussion}

Considerable clinical and experimental evidence exists to indicate that patients with sepsis develop a state of immunoparalysis during the evolution of the disease, underscoring the complexity of the host response to sepsis. Early in sepsis, a vigorous proinflammatory response occurs, which contributes to the multisystem organ failure and mortality of systemic inflammatory response syndrome (SIRS). It is also apparent that septic insults initiate concurrent or sequential antiinflammatory cascades during the course of sepsis, which likely mitigates the extent of inflammation and tissue injury. This antiinflammatory response, referred to as compensatory antiinflammatory response syndrome (CARS), is also believed to contribute to the increased susceptibility of critically ill patients to secondary nosocomial infections, due in part to sepsis-induced leukocyte "immunoparalysis" $(2,5,8,11$, $32-36)$. Interestingly, mortality is higher in patients with sepsis who display evidence of monocyte deactivation (2). Furthermore, monocytes isolated from patients who went on to survive their septic event were found to regain LPS responsiveness (2).

At present, the molecular mechanisms underlying the immunoparalysis of sepsis remain poorly characterized. In this study, we shed some light on the importance of the LPS/TLR4 signaling axis in mediating sepsis-induced immunosuppression among animals rendered septic by CLP. In particular, we observed that the negative regulator of TLR signaling, IRAK-M, is upregulated in macrophages of the lung during sepsis and plays a role in mediating the hyporesponsiveness of these macrophages to LPS. Our results support earlier published studies that demonstrated prolonged or repeated exposure to TLR ligands (e.g., LPS, peptidoglycan) or other virulence factors such as Mycobacterial ManLAM in vitro led to the induction of IRAK-M expression $(19,20,37)$. IRAK-M was shown to inhibit NF- $\mathrm{KB}$ activation and downregulate inflammatory cytokine production in macrophages rendered tive innate immunity against bacterial pathogens of the lung. Our studies demonstrate that lung macrophages from animals deficient in IRAK-M display a relative resistance to the development of sepsis-induced LPS hyporesponsiveness, as evidenced by their ability to express proinflammatory cytokines such as IL-12 and TNF- $\alpha$ when stimulated with LPS ex vivo. Furthermore, we observed that macrophages from IRAK-M-deficient mice undergoing CLP retained the ability to express the costimulatory molecules CD40 and CD86 in response to intrapulmonary bacterial administration. Finally, septic IRAK-M-1- mice exhibited a more robust production of inflammatory cytokines in the lung when challenged with $P$. aeruginosa, as compared with the blunted cytokine response observed in similarly treated WT mice. It is notable that the expression of IRAK-M is believed to be limited to cells of monocytic lineage, as other leukocyte populations have not been shown to express this protein under various in vitro experimental conditions $(17,18)$. It remains a possibility that IRAK-M may be induced in other cell types (e.g., neutrophils and/ or dendritic cells) during periods of physiologic stress in vivo, although this has not yet been demonstrated. Therefore, these data taken together suggest that the dampened lung inflammatory response observed in septic mice is at least partially attributable to altered macrophage function.

We demonstrate that IRAK-M plays an important role in regulating the generation of protective innate immune responses in the lung in vivo. An earlier study examining the role of IRAK-M in vivo found that IRAK-M $M^{-/-}$mice had markedly increased inflammatory enteric responses when orally infected with Salmonella typhimurium (19). In our study, we observed that the absence of IRAK-M led to the enhancement of bacterial clearance from the lung and blood by preserving macrophage effector function during sepsis, resulting in the more vigorous recruitment (and perhaps activation) of neutrophils to the pulmonary airspaces via the production of neutrophil-active cytokines and chemokines. While IRAK-M has clearly been shown to inhibit LPS/TLR4-mediated inflammatory responses in macrophages, we cannot exclude the possibility that IRAK-M may regulate other important antimicrobial functions of macrophages independent of effects on TLR signaling. However, 
we did not find any differences in phagocytic or bactericidal activity of macrophages isolated from septic IRAK-M $M^{-/-}$mice, as compared with their septic WT counterparts (data not shown).

Other mediators of endotoxin tolerance and/or sepsis-induced macrophage deactivation are poorly defined. Candidate molecules/events include changes in cell-surface TLR expression, interruption of downstream TLR signaling cascades, or direct suppressive effects of antiinflammatory molecules that are released during sepsis. Of note, we did not observe appreciable differences in the expression of TLR2, TLR4, or CD14 in whole lung or on the surface of alveolar macrophages from CLP mice as compared with AMs from sham-operated animals. Furthermore, unlike macrophages rendered "endotoxin tolerant" in vitro, alveolar macrophages from septic mice and sham-operated controls appeared to have comparable expression of IRAK-1 and IRAK-4, demonstrating that the mechanism of sepsis-induced LPS hyporesponsiveness is independent of IRAK-1 and IRAK-4 expression. Antiinflammatory cytokines such as IL-10, IL-4, IL-13, and TGF- $\beta$ and non-cytokine mediators, including prostaglandin $\mathrm{E}_{2}$, catecholamines, and glucocorticoids, have been detected in the serum of patients and/or experimental animals with sepsis (38-42). In particular, IL-10 appears to contribute to sepsis-induced inhibition of macrophage responses $(10,21,43)$. In our study, however, we found a trend toward increased IL-10 production in IRAK-M $M^{-/-}$ animals following CLP and i.t. Pseudomonas infection, demonstrating that differences in IL-10 production are not responsible for the relative preservation of pulmonary innate immune responses in septic IRAK-M-1- mice.

In addition to IRAK-M, many negative regulators of TLR signaling have been identified, including the TLR homolog RP105, Src homology 2-containing inositol-5'-phosphatase (SHIP), short form of MyD88, ST2, SIGIRR, Triad3A, A20, and SOCS-1 (44-52). Many of these molecules have been implicated in the development of endotoxin tolerance. In addition, negative regulators of NF- $\kappa \mathrm{B}$ activation, such as Ran GTPase, A20 binding inhibitor of NF- $\kappa$ B activation-1 and -2 (ABIN-1 and -2), and dual specificity phosphatase 1 (DUSP1), have been shown to limit inflammatory gene expression, including during experimental endotoxininduced shock (53-57). It is expected that multiple inhibitory pathways are activated during the course of sepsis that function to limit the magnitude of macrophage-driven inflammation. Furthermore, these inhibitory molecules may also contribute to sepsis-induced immune dysfunction of other cell types, such as neutrophils or epithelial cells. For example, SIGIRR is expressed primarily by epithelial cells, and its expression serves to dampen LPS-induced activation of these cells. Even though it was not as pronounced as that observed in WT mice, we did observe a trend toward reduced cytokine production from LPS-stimulated AMs isolated from septic IRAK- $M^{-/-}$animals, as compared with cells recovered from $I R A K-M^{-/-}$mice undergoing sham surgery, suggesting that this partially deactivated AM phenotype is mediated by inhibitory molecules other than IRAK-M. Nonetheless, the marked induction of IRAK-M, combined with the relative resistance of IRAK-M-knockout mice to developing macrophage deactivation and impaired antibacterial immunity during sepsis, indicate that this molecule is a major mediator of sepsis-induced macrophage dysfunction in this setting.

Signals that trigger the expression of IRAK-M mRNA and protein by AMs in CLP have not been well characterized. Resting monocytes or tissue macrophages express very little IRAK-M message or protein constitutively. However, we and others have shown that LPS, peptidoglycan, and other PAMPs can stimulate macrophage IRAK-M expression (19, 20, 23, 37). Moreover, we have found that certain host-derived molecules released in sepsis can drive the synthesis of IRAK-M. In particular, the treatment of a murine alveolar macrophage cell line (MH-S) with TNF- $\alpha$ in vitro induces IRAK-M mRNA expression, albeit to a lesser degree than LPS stimulation (data not shown). In contrast, we have not detected induction of IRAK-M after in vitro incubation of MH-S cells with selected antiinflammatory cytokines, including IL-10 (data not shown). The induction of IRAK-M by host-derived factors raises the distinct possibility that this molecule may contribute to macrophage dysfunction in disease states that are not initiated by microbial pathogens.

IRAK-M is ideally positioned to regulate macrophage function in response to infectious challenge. This molecule can function to dampen macrophage responses to multiple PAMPs in sepsis, thereby limiting the release of injurious host-derived molecules. However, by impairing PAMP recognition and signaling during the septic response, this protein can also exert profound suppressive effects on the generation of protective innate responses. Therefore, targeting of IRAK-M during the various phases of sepsis is likely to have a substantial impact on the course of this disease. Because TLRs are critical receptors in the recognition of and response to microbial pathogens, the neutralization of IRAK-M under conditions where this protein is induced (e.g., sepsis) might allow the septic host to mount a more effective host response to pathogens responsible for inducing sepsis or nosocomial pathogens encountered during the peri-sepsis period. Future studies will focus on defining the contribution of IRAK-M to sepsis-induced immunosuppression following secondary challenge with other clinically relevant nosocomial pathogens, including fungal (e.g., Candida) and gram-positive bacteria.

\section{Methods}

Reagents. Recombinant murine anti-TNF- $\alpha,-$ MIP-2, -IL-12p70, -IP-10, and -IL-10 antibodies used in the ELISAs were purchased from R\&D Systems. Anti-IRAK protein antibodies used in Western immunoblotting were purchased from Chemicon International (IRAK-M) and Cell Signaling Technology (IRAK-1 and IRAK-4). The LPS used was ion-exchange chromatography-purified E. coli LPS O55:B5 from Sigma-Aldrich.

Mice. A colony of IRAK-M-deficient mice bred on a B6 background were established at the University of Michigan. Age- and sex-matched specific pathogen-free 6- to 8-week-old C57BL/6J mice were purchased from the Jackson Laboratory. All animals were housed in specific pathogen-free conditions within the University of Michigan animal care facility (Ann Arbor, Michigan, USA) until the day of sacrifice. All animal experiments were performed in accordance with NIH policies on the humane care and use of laboratory animals and approved by the University Committee on Use and Care of Animals (UCUCA) at the University of Michigan.

CLP. CLP was used as a model of systemic sepsis syndrome as previously described (58). Pathogen-free C57BL/6 mice were anesthetized with a mixture of i.p. xylazine and ketamine. A $1-\mathrm{cm}$ longitudinal incision was made to the lower-right quadrant of the abdomen and the cecum exposed. The distal one-third of the cecum was ligated with a 3-0 silk suture and punctured through with a 26-gauge needle. Puncture with this gauge needle results in a marked septic response but with death occurring in $0 \%-10 \%$ of animals. The cecum was then placed back in the peritoneal cavity, and the incision was closed with surgical staples. In sham surgical controls, the cecum was exposed but not 
ligated or punctured, then returned to the abdominal cavity. All mice were administered $1 \mathrm{ml}$ of sterile saline s.c. for fluid resuscitation during the immediate postoperative period.

Bacterial preparation and i.t. inoculation. P. aeruginosa (UI-18 strain; Pfizer) was used in our studies. Bacteria were grown overnight in tryptic soy broth (Difco; BD Diagnostics) at $37^{\circ} \mathrm{C}$ while constantly shaken. The concentration of bacteria in broth was determined by measuring the absorbance at $600 \mathrm{~nm}$ and then plotting the OD on a standard curve generated by known CFU values. The bacteria culture was then diluted to the desired concentration. Mice were anesthetized with an i.p. ketamine and xylazine mixture. Next, the trachea was exposed, and $30 \mu \mathrm{l}$ of inoculum was administered via a sterile 26 -gauge needle. The skin incision was closed using surgical staples.

Whole-lung homogenization for determination of CFU. At designated time points, the mice were euthanized by $\mathrm{CO}_{2}$ inhalation. Before lung removal, the pulmonary vasculature was perfused by infusing $1 \mathrm{ml}$ of PBS containing $5 \mathrm{mM}$ EDTA into the right ventricle. Whole lungs were removed, with care taken to dissect away lymph nodes. The lungs were then homogenized in $1 \mathrm{ml}$ of PBS with protease inhibitor (Roche Diagnostics). Homogenates were then serially diluted 1:5 in PBS and plated on blood agar to determine lung CFU.

Peripheral blood CFU determination. Blood was collected in a heparinized syringe from the right ventricle at 24 hours following i.t. Psendomonas administration, serially diluted 1:2 with PBS, and plated on blood agar to determine blood CFU.

$B A L$. BAL was performed for assessment of leukocyte recruitment and to obtain airspace leukocytes for ex vivo studies. The trachea was exposed and intubated using a $1.7-\mathrm{mm}$-outer-diameter polyethylene catheter. BAL was performed by instilling PBS containing $5 \mathrm{mM}$ EDTA in 1-ml aliquots. One or $10 \mathrm{ml}$ of PBS was instilled per mouse, with $7 \mathrm{ml}$ of lavage fluid retrieved. Lavaged cells from each group of animals were pooled and counted after lysis with hypotonic solution. Cytospins (Thermo Electron Corp.) were prepared for determination of BAL differentials using a modified Wright stain. Lavaged cells consisted of more than $95 \%$ AMs for each of the groups examined (data not shown). Cells were resuspended in RPMI 1640 medium (Invitrogen) to give a concentration of 500,000 cells $/ \mathrm{ml}$. 250,000 cells were placed in each well of a 24-well cell culture plate (Corning Inc.) and washed with $500 \mu$ l of RPMI 1640 after 45 minutes, enabling the isolation of AMs by adherence. LPS was then added to selected wells and incubated for 16 hours. Supernatants were collected and the production of TNF- $\alpha$ and IL-12 determined by ELISA.

Total lung leukocyte preparation by lung digest. Lungs were removed from euthanized animals and leukocytes prepared as previously described (26). Briefly, lungs were minced with scissors to fine slurry in $15 \mathrm{ml}$ of digestion buffer (RPMI, $10 \%$ fetal calf serum, $1 \mathrm{mg} / \mathrm{ml}$ collagenase [Roche Diagnostics], $30 \mu \mathrm{g} / \mathrm{ml}$ DNase [Sigma-Aldrich]) per lung. Lung slurries were enzymatically digested for 30 minutes at $37^{\circ} \mathrm{C}$. Any undigested fragments were further dispersed by drawing the solution up and down through the bore of a 10- $\mathrm{ml}$ syringe. The total lung cell suspension was pelleted, resuspended, and spun through a $40 \%$ Percoll gradient to enrich for leukocytes. Cell counts and viability were determined using Trypan blue exclusion counting on a hemacytometer. Cytospin slides were prepared and stained with a modified Wright-Giemsa stain.

MPO assay. Lung MPO activity (as an assessment of neutrophil influx) was quantitated using a previously described method (59). Briefly, whole lungs were homogenized in $2 \mathrm{ml}$ of a solution containing $50 \mathrm{mM}$ potassium phosphate, $\mathrm{pH} 6.0$, with $5 \%$ hexadecyltrimethylammonium bromide and $5 \mathrm{mM}$ EDTA. The resulting homogenate was sonicated and centrifuged at $12,000 \mathrm{~g}$ for 15 minutes. The supernatant was mixed 1:15 with assay buffer and read at $490 \mathrm{~nm}$. MPO units were calculated as the change in absorbance over time.
Murine cytokine ELISA. Murine IL-12p70, TNF- $\alpha$, MIP-2, IP-10, and IL-10 were quantitated using a modification of a double ligand method as previously described (13). A standard curve was generated using serial half-log dilutions of murine recombinant cytokines from $1 \mathrm{pg} / \mathrm{ml}$ to $100 \mathrm{ng} / \mathrm{ml}$. This ELISA method consistently detected murine cytokine concentrations higher than $50 \mathrm{pg} / \mathrm{ml}$. The ELISAs did not cross-react with other cytokines tested.

Isolation and RT-PCR amplification of whole-lung $m R N A$. Whole lung was harvested, immediately snap-frozen in liquid nitrogen, and stored at $-70^{\circ} \mathrm{C}$. RNA was isolated, and RT-PCR was performed as previously described (13). Briefly, total cellular RNA from the frozen lungs was isolated, reversed transcribed into cDNA, and then amplified using specific primers for mTLR2 and mTLR4, with $\beta$-actin serving as a control. The primer sequences for mTLR2 were forward 5'-CAGCTTAAAGGGGGGGTCAGAG-3', reverse 5'-TGGAGACGCCAGCTCTGGCTCA-3'; for mTLR4, forward 5'-AGCGGGTCAAGGAACAGAAGCA-3', reverse 5'-CTTTACCAGCTCATTTCTCACC-3'; for $\beta$-actin $5^{\prime}$-ATGGATGACGATATCGCTC- $3^{\prime}$ and $5^{\prime}$-GATTCCATACCCAGGAGGG-3'. After amplification (35 cycles for TLR2 and TLR4, 25 cycles for $\beta$-actin), the samples $(20 \mu \mathrm{l})$ were separated on a $2 \%$ agarose gel containing $5 \mu \mathrm{l} / 100 \mathrm{ml}$ ethidium bromide (Sigma-Aldrich), and bands were visualized and photographed using UV transillumination.

Real-time quantitative RT-PCR. Measurement of gene expression was performed utilizing the ABI Prism 7000 Sequence Detection System (Applied Biosystems) as previously described (27). Briefly, primer and probe for $\beta$ actin and IP-10 were designed using Primer Express software (Applied Biosystems). The primers, placed in different exons, were tested to ensure that they do not amplify genomic DNA. Primers and probe nucleotide sequences for mIP-10 were as follows: forward primer 5'-CCAGTGAGAATGAGGGCCATA-3', reverse primer 5'-CTCAACACGTGGGCAGGAT-3', TaqMan probe $5^{\prime}$ (FAM)-TTTGGGCATCATCTCCTGGA-(TAMR) 3'; for mTNF- $\alpha$ : forward 5'-CAGCCGATGGGTTGTACCTT-3', reverse 5'-TGTGGGTGAGGAGCACGTAGT-3', probe 5'-TCCCAGGTTCTCTTCAAGGGACAAGGC3'; for mMIP-2: forward 5'-GAACATCCAGAGCTTGAGTGTGA-3', reverse 5'-CCTTGAGAGTGGCTATGACTTCTGT-3', probe 5'-CCCCCAGGACCCCACTGCG-3'; for mIL-12p40: forward 5'-AGACCCTGCCCATTGAACTG-3', reverse 5'-GAAGCTGGTGCTGTAGTTCTCATATT-3', probe 5'-CGTTGGAAGCACGGCAGCAGAA-3'; for mIRAK-M: forward 5'-TGAGCAACGGGACGCTTT-3', reverse 5'-GATTCGAACGTGCCAGGAA-3', probe $5^{\prime}$-TTACAGTGCACAAATGGCACAACCCC-3'; for m $\beta$-actin: forward 5'-CCGTGAAAAGATGACCCAGATC-3', reverse 5'-CACAGCCTGGATGGCTACGT-3', probe 5'-TTTGAGACCTTCAACACCCCAGCCA-3'. Specific thermal cycling parameters used with the TaqMan One-Step RT-PCR Master Mix Reagents kit (Applied Biosystems; included 30 minutes at $48^{\circ} \mathrm{C}$, 10 minutes at $95^{\circ} \mathrm{C}$, and 40 cycles involving denaturation at $95^{\circ} \mathrm{C}$ for 15 seconds, annealing/extension at $60^{\circ} \mathrm{C}$ for 1 minute. Relative quantitation of cytokine mRNA levels was plotted as fold change relative to untreated control cells of the lung. All experiments were performed in duplicate.

Multiparameter flow-cytometric analyses. Cells were isolated from BAL or lung digests as described above. At 24 hours following CLP or sham surgery alone, lavaged cells were less than $95 \%$ AMs in morphology. These cells were stained with anti-TLR2, anti-TLR4, and anti-CD14 (eBioscience) for determination of TLR expression. BAL cells were also recovered from sham-operated or CLP mice at time 0 and 6 hours after $P$. aeruginosa administration, gated for macrophage populations, and stained with anti-CD40, anti-CD80, and anti-CD86 (BD Biosciences - Pharmingen). For analyses of $\mathrm{T}$ cell subsets, isolated leukocytes from lung digest were stained with the following FITC- or PE-labeled antibodies: anti- $\gamma \delta$ TCR, anti- $\alpha \beta$ TCR, anti-CD8, anti-CD4, anti-DX5, and anti-CD 69 (BD Biosciences - Pharmingen). In addition, cells were stained with anti-CD45Tricolor (Invitrogen), allowing for discrimination of leukocytes from non-leukocytes and thus eliminating any nonspecific binding of $\mathrm{T}$ cell 
surface markers on non-leukocytes. T and NK cell subsets were analyzed first by gating on CD45-positive "lymphocyte-sized" leukocytes and then examined for FL1 and FL2 fluorescence expression. Cells were collected on a FACScan or FACSCalibur Flow Cytometer (BD Biosciences) using CellQuest software version $3.2(\mathrm{BD})$. Analyses of data were performed using the CellQuest software package.

Western immunoblotting. Pulmonary macrophages were isolated by adherence purification of leukocytes obtained from whole-lung digest as described above. Whole-cell lysates were obtained by treating cells with RIPA buffer (1\% wt/wt NP-40, 1\% wt/vol sodium deoxycholate, $0.1 \% \mathrm{wt} / \mathrm{vol}$ SDS, $0.15 \mathrm{M} \mathrm{NaCl}, 0.01 \mathrm{M}$ sodium phosphate, $2 \mathrm{mM}$ EDTA, and $50 \mathrm{mM}$ sodium fluoride) plus protease and phosphatase inhibitors (Protease Inhibitor Cocktail [Sigma-Aldrich] and sodium orthovanadate, respectively). Protein concentrations were determined by the Bio-Rad DC Protein Assay. Samples were electrophoresed in 4\%-12\% gradient SDS-PAGE gels, transferred to nitrocellulose, and blocked with 5\% skim milk in PBS. After incubation with primary antibodies, blots were incubated with secondary antibody linked to HRP, and bands visualized using enhanced chemiluminescence (SuperSignal West Pico Substrate; Pierce Biotechnology).

Phagocytosis assay. Alveolar macrophages were isolated from BAL fluid and plated at a density of 300,000 macrophages/well on a half-area, black 96-well tissue culture plate. After 1 hour of incubation at $37^{\circ} \mathrm{C}$, cell supernatant was removed, and the plates were inspected to ensure that the wells had greater than $75 \%$ confluence and were approximately equal in cell density. Fifty microliters of fluorescently labeled E. coli bioparticles (Vybrant Phagocytosis Assay; Invitrogen) were added to each well at a ratio of 60 bacteria/ 1 cell, and the plates were incubated for 2 hours at $37^{\circ} \mathrm{C}$. Trypan blue dye was added to each well to quench the fluorescence of extracellular bacteria, and intracellular bacteria were quantitated by fluorimetry as described previously (60).

Tetrazolium dye reduction bactericidal assay. This assay was performed as described previously (61). Briefly, alveolar macrophages were isolated from BAL fluid from septic WT and IRAK-M $M^{-/-}$animals and counted and assessed for viability using Trypan blue exclusion. AMs were plated at a density of $10^{6}$ viable cells/well in duplicate on 96-well tissue culture plates and adherence purified for 1 hour. Cells were then incubated with a $0.1-\mathrm{ml}$ suspension of opsonized E. coli $\left(10^{7} \mathrm{CFU} / \mathrm{ml}\right.$; MOI, 50:1) for 30 minutes to allow phagocytosis to occur. The cell monolayers were washed of unbound bacteria with PBS twice. Twenty microliters of tetrazolium dye stock solution was added to all wells, and plates were shaken gently for 15 minutes at $37^{\circ} \mathrm{C}$. The intensity of the absorbance at $595 \mathrm{~nm}$ was measured, which is directly proportional to the number of intracellular bacteria associated with the macrophages.

Statistics. Survival curves were compared using the log-rank test. For other data, statistical significance was determined using the unpaired 2 -tailed Student's $t$ test or 1-way ANOVA corrected for multiple comparisons where appropriate. $P$ values less than 0.05 were considered statistically significant. All calculations were performed using the Prism 3.0 software program for Windows (GraphPad Software).

\section{Acknowledgments}

This work was supported by NIH grants K08HL081229 (to J.C. Deng) and PO50 HL60289 and HL57243 (to T.J. Standiford). R.A. Flavell is an investigator with the Howard Hughes Medical Institute.

Received for publication January 26, 2006, and accepted in revised form June 27, 2006.

Address correspondence to: Jane C. Deng, Department of Medicine, Division of Pulmonary and Critical Care Medicine, David Geffen School of Medicine at UCLA, 10833 LeConte Avenue, 37-131 CHS, Los Angeles, California 90095, USA. Phone: (310) 825-0930; Fax: (310) 206-8622; E-mail: jdeng@mednet.ucla.edu.
1. Jacobs, R.F., Kiel, D.P., and Balk, R.A. 1986. Alveolar macrophage function in a canine model of endotoxin-induced lung injury. Am. Rev. Respir. Dis. 134:745-751.

2. Munoz, C., et al. 1991. Dysregulation of in vitro cytokine production by monocytes during sepsis. J. Clin. Invest. 88:1747-1754.

3. Nelson, S., Chidiac, C., Bagby, G., and Summer, W.R. 1990. Endotoxin-induced suppression of lung host defenses. J. Med. 21:85-103.

4. Volk, H.D., et al. 1996. Monocyte deactivation - rationale for a new therapeutic strategy in sepsis. Intensive Care Med. 22(Suppl. 4):S474-S481.

5. Volk, H.D., et al. 1993. Alterations in function and phenotype of monocytes from patients with septic disease: predictive value and new therapeutic strategies. In Host defense dysfunction in trauma, shock, and sepsis. E. Faist, J.L. Meakins, and F.W. Schildberg, editors. Springer-Verlag. Berlin/Heidelberg, Germany. 365-371.

6. Broug-Holub, E., et al. 1997. Alveolar macrophages are required for protective pulmonary defenses in murine Klebsiella pneumonia: elimination of alveolar macrophages increases neutrophil recruitment but decreases bacterial clearance and survival. Infect. Immun. 65:1139-1146.

7. Chen, G.H., et al. 2000. Intrapulmonary TNF gene therapy reverses sepsis-induced suppression of lung antibacterial host defense. J. Immunol. 165:6496-6503.

8. Docke, W.D., et al. 1997. Monocyte deactivation in septic patients: restoration by IFN-gamma treatment. Nat. Med. 3:678-681.

9. Reddy, R.C., Chen, G.H., Tekchandani, P.K., and Standiford, T.J. 2001. Sepsis-induced immunosuppression: from bad to worse. Immunol. Res. 24:273-287.
10. Steinhauser, M.L., et al. 1999. IL-10 is a major mediator of sepsis-induced impairment in lung antibacterial host defense. J. Immunol. 162:392-399.

11. Volk, H.D., et al. 1991. Alterations in function and phenotype of monocytes from patients with septic disease - predictive value and new therapeutic strategies. Behring. Inst. Mitt. 11:208-215.

12. Deng, J.C., Tateda, K., Zeng, X., and Standiford, T.J. 2001. Transient transgenic expression of gamma interferon promotes Legionella pneumophila clearance in immunocompetent hosts. Infect. Immun. 69:6382-6390.

13. Greenberger, M.J., et al. 1996. IL-12 gene therapy protects mice in lethal Klebsiella pneumonia. J. Immunol. 157:3006-3012.

14. Laichalk, L.L., et al. 1996. Tumor necrosis factor mediates lung antibacterial host defense in murine Klebsiella pneumonia. Infect. Immun. 64:5211-5218.

15. Standiford, T.J., et al. 1999. Intrapulmonary tumor necrosis factor gene therapy increases bacterial clearance and survival in murine gram-negative pneumonia. Hum. Gene Ther. 10:899-909.

16. Deng, J.C., et al. 2004. CpG oligodeoxynucleotides stimulate protective innate immunity against pulmonary Klebsiella infection. J. Immunol. 173:5148-5155.

17. Rosati, O., and Martin, M.U. 2002. Identification and characterization of murine IRAK-M. Biochem. Biophys. Res. Commun. 293:1472-1477.

18. Wesche, H., et al. 1999. IRAK-M is a novel member of the Pelle/interleukin-1 receptor-associated kinase (IRAK) family. J. Biol. Chem. 274:19403-19410.

19. Kobayashi, K., et al. 2002. IRAK-M is a negative regulator of Toll-like receptor signaling. Cell. 110:191-202.

20. Nakayama, K., et al. 2004. Involvement of IRAK-M in peptidoglycan-induced tolerance in macrophages. J. Biol. Chem. 279:6629-6634.
21. Reddy, R.C., et al. 2001. Alveolar macrophage deactivation in murine septic peritonitis: role of interleukin 10. Infect. Immun. 69:1394-1401.

22. Adib-Conquy, M., et al. 2000. NF-kappaB expression in mononuclear cells of patients with sepsis resembles that observed in lipopolysaccharide tolerance. Am. J. Respir. Crit. Care Med. 162:1877-1883.

23. Escoll, P., et al. 2003. Rapid up-regulation of IRAK-M expression following a second endotoxin challenge in human monocytes and in monocytes isolated from septic patients. Biochem. Biophys. Res. Commun. 311:465-472.

24. Li, L., Cousart, S., Hu, J., and McCall, C.E. 2000. Characterization of interleukin-1 receptor-associated kinase in normal and endotoxin-tolerant cells. J. Biol. Chem. 275:23340-23345.

25. Hatao, F., et al. 2004. Prolonged Toll-like receptor stimulation leads to down-regulation of IRAK-4 protein. J. Leukoc. Biol. 76:904-908.

26. Tsai, W.C., et al. 2000. CXC chemokine receptor CXCR2 is essential for protective innate host response in murine Pseudomonas aeruginosa pneumonia. Infect. Immun. 68:4289-4296.

27. Zeng, X., et al. 2003. Intrapulmonary expression of macrophage inflammatory protein 1alpha (CCL3) induces neutrophil and NK cell accumulation and stimulates innate immunity in murine bacterial pneumonia. Infect. Immun. 71:1306-1315.

28. Zeng, X., et al. 2005. Interferon-inducible protein 10 , but not monokine induced by gamma interferon, promotes protective type 1 immunity in murine Klebsiella pneumoniae pneumonia. Infect. Immun. 73:8226-8236.

29. Tateda, K., et al. 2001. Chemokine-dependent neutrophil recruitment in a murine model of Legionella pneumonia: potential role of neutrophils as immunoregulatory cells. Infect. Immun. 69:2017-2024. 
30. Chen, S.C., et al. 2001. Impaired pulmonary host defense in mice lacking expression of the $\mathrm{CXC}$ chemokine lungkine. J. Immunol. 166:3362-3368.

31. Tsai, W.C., et al. 1998. Lung-specific transgenic expression of $\mathrm{KC}$ enhances resistance to Klebsiella pneumoniae in mice. J. Immunol. 161:2435-2440.

32. Bone, R.C. 1996. Immunologic dissonance: a continuing evolution in our understanding of the systemic inflammatory response syndrome (SIRS) and the multiple organ dysfunction syndrome (MODS). Ann. Intern. Med. 125:680-687.

33. Marie, C., et al. 1998. Reduced ex vivo interleukin-8 production by neutrophils in septic and nonseptic systemic inflammatory response syndrome. Blood. 91:3439-3446.

34. Carreras, M.C., Catz, S.D., Pargament, G.A., Del Bosco, C.G., and Poderoso, J.J. 1994. Decreased production of nitric oxide by human neutrophils during septic multiple organ dysfunction syndrome. Comparison with endotoxin and cytokine effects on normal cells. Inflammation. 18:151-161.

35. McCall, C.E., Grosso-Wilmoth, L.M., LaRue, K., Guzman, R.N., and Cousart, S.L. 1993. Tolerance to endotoxin-induced expression of the interleukin-1 $\beta$ gene in blood neutrophils of humans with the sepsis syndrome. J. Clin. Invest. 91:853-861.

36. Simpson, S.Q., Modi, H.N., Balk, R.A., Bone, R.C., and Casey, L.C. 1991. Reduced alveolar macrophage production of tumor necrosis factor during sepsis in mice and men. Crit. Care Med. 19:1060-1066.

37. Pathak, S.K., et al. 2005. Mycobacterium tuberculosis lipoarabinomannan-mediated IRAK-M induction negatively regulates Toll-like receptor-dependent interleukin-12 p40 production in macrophages. J. Biol. Chem. 280:42794-42800.

38. Junger, W.G., Hoyt, D.B., Liu, F.C., Loomis, W.H., and Coimbra, R. 1996. Immunosuppression after endotoxin shock: the result of multiple antiinflammatory factors. J. Trauma. 40:702-709.

39. Marie, C., Cavaillon, J.M., and Losser, M.R. 1996. Elevated levels of circulating transforming growth factor-beta 1 in patients with the sepsis syndrome. Ann. Intern. Med. 125:520-521.

40. Collighan, N., et al. 2004. Interleukin 13 and inflammatory markers in human sepsis. Br. J. Surg. 91:762-768.

41. Choudhry, M.A., Ahmad, S., and Sayeed, M.M. 1995. Role of $\mathrm{Ca} 2+$ in prostaglandin E2-induced T-lymphocyte proliferative suppression in sepsis. Infect. Immun. 63:3101-3105.

42. Jones, S.B., Westfall, M.V., and Sayeed, M.M. 1988 Plasma catecholamines during E. coli bacteremia in conscious rats. Am. J. Physiol. 254:R470-R477.

43. Fumeaux, T., and Pugin, J. 2002. Role of interleukin-10 in the intracellular sequestration of human leukocyte antigen-DR in monocytes during septic shock. Am. J. Respir. Crit. Care Med. 166:1475-1482.

44. Kinjyo, I., et al. 2002. SOCS1/JAB is a negative regulator of LPS-induced macrophage activation. Immunity. 17:583-591.

45. Boone, D.L., et al. 2004. The ubiquitin-modifying enzyme A20 is required for termination of Toll-like receptor responses. Nat. Immunol. 5:1052-1060

46. Wertz, I.E., et al. 2004. De-ubiquitination and ubiquitin ligase domains of A20 downregulate NF-kappaB signalling. Nature. 430:694-699.

47. Nakagawa, R., et al. 2002. SOCS-1 participates in negative regulation of LPS responses. Immunity. 17:677-687.

48. Rauh, M.J., et al. 2005. SHIP represses the generation of alternatively activated macrophages. Immunity. 23:361-374.

49. Sly, L.M., Rauh, M.J., Kalesnikoff, J., Song, C.H., and Krystal, G. 2004. LPS-induced upregulation of SHIP is essential for endotoxin tolerance. Immunity. 21:227-239.

50. Divanovic, S., et al. 2005. Negative regulation of Toll-like receptor 4 signaling by the Toll-like receptor homolog RP105. Nat. Immunol. 6:571-578.

51. Chuang, T.H., and Ulevitch, R.J. 2004. Triad3A, an E3 ubiquitin-protein ligase regulating Toll-like receptors. Nat. Immunol. 5:495-502.
52. Brint, E.K., et al. 2004. ST2 is an inhibitor of interleukin 1 receptor and Toll-like receptor 4 signaling and maintains endotoxin tolerance. Nat. Immunol. 5:373-379.

53. Heyninck, K., et al. 1999. The zinc finger protein A20 inhibits TNF-induced NF-kappaB-dependent gene expression by interfering with an RIP- or TRAF2-mediated transactivation signal and directly binds to a novel NF-kappaB-inhibiting protein ABIN. J. Cell Biol. 145:1471-1482.

54. Van Huffel, S., Delaei, F., Heyninck, K., De Valck, D., and Beyaert, R. 2001. Identification of a novel A20-binding inhibitor of nuclear factorkappa B activation termed ABIN-2. J. Biol. Chem. 276:30216-30223.

55. Hammer, M., et al. 2006. Dual specificity phosphatase 1 (DUSP1) regulates a subset of LPSinduced genes and protects mice from lethal endotoxin shock. J. Exp. Med. 203:15-20.

56. Wong, P.M., and Chung, S.W. 2003. A functional connection between RanGTP, NF-kappaB and septic shock. J. Biomed. Sci. 10:468-474.

57. Wong, P.M., et al. 1999. Lps(d)/Ran of endotoxinresistant $\mathrm{C} 3 \mathrm{H} / \mathrm{HeJ}$ mice is defective in mediating lipopolysaccharide endotoxin responses. Proc. Natl. Acad. Sci. U. S. A. 96:11543-11548.

58. Walley, K.R., Lukacs, N.W., Standiford, T.J., Strieter, R.M., and Kunkel, S.L. 1996. Balance of inflammatory cytokines related to severity and mortality of murine sepsis. Infect. Immun. 64:4733-4738.

59. Goldblum, S.E., Wu, K.M., and Jay, M. 1985. Lung myeloperoxidase as a measure of pulmonary leukostasis in rabbits. J. Appl. Physiol. 59:1978-1985.

60. Ojielo, C.I., et al. 2003. Defective phagocytosis and clearance of Pseudomonas aeruginosa in the lung following bone marrow transplantation. J. Immunol. 171:4416-4424.

61. Ofek, I., et al. 2001. Surfactant protein D enhances phagocytosis and killing of unencapsulated phase variants of Klebsiella pneumoniae. Infect. Immun. 69:24-33 